\title{
Pulsatile flow analysis in a bend square channel by modal decomposition
}

\author{
Lukáš Manoch $^{1, a}$, Jan Matěcha ${ }^{1, b}$, Hana Netřebská ${ }^{1, c}$, and Josef Adamec ${ }^{1, d}$ \\ CTU in Prage, Faculty of Mechanical Engineering, Department of Fluid Dynamics and Thermodynamics, Technická 4, \\ Praha 6, Czech Republic
}

\begin{abstract}
The article analyzes the results of numerical simulations of pulsatile flow in a square channel with a $90^{\circ}$ bend using Proper Orthogonal Decomposition (POD). The simulations of laminar steady and unsteady flow obtained by ANSYS Fluent commercial system were used as input for modal decomposition. Several regimes for different values of individual parameters (stationary flow component, ) were simulated. Not only energy-related modes but also modes significant from the viewpoint of energy dissipation. Other evaluated modes are the modes corresponding to the pulsatile flow frequency and the modes reflecting the flow separation at the bend.
\end{abstract}

\section{Aim}

The aim of the paper was to analyze the results of numerical simulation of unsteady pulsatile flow in the square cross-section channel with a $90^{\circ}$ bend in the middle of its length using the Proper Orthogonal Decomposition (POD) method.

\section{Model}

The geometric model of channel for numerical simulation was created within the Designer program, which is part of the ANSYS Workbench v14. The channel model had a constant square cross-section over the whole length with an edge length of $5 \mathrm{~mm}$. The channel was, in its whole length of $1000 \mathrm{~mm}$, divided into several independent, consecutive sections. The lengths of the sections at the inlet and outlet from the computational area were $250 \mathrm{~mm}$ and they could be described as input (section 1) / output (section 5), or stabilizing sections. The remaining internal sections were considered to be the sections suitable for flow parameters evaluation. The middle section (3) out of 5 sections - had dimensions of a cube with a $5 \mathrm{~mm}$ edge. Such dimensionally designed channel allowed the use of the same geometry even for data verification in a straight channel. The remaining two, yet unspecified sections, namely section 2 and 4, were divided proportionally. All the aforementioned divisions and constructions were based on [1].

\section{Numerical simulation}

\subsection{Computational mesh}

For the created channel a structured mesh containing only elements of six-wall type was defined. A non-uniform mesh

\footnotetext{
a e-mail: lukas.manoch@fs.cvut.cz

b e-mail: jan.matecha@fs.cvut.cz

c e-mail: hana.netrebska@fs.cvut.cz

d e-mail: josef.adamec@fs.cvut.cz
}

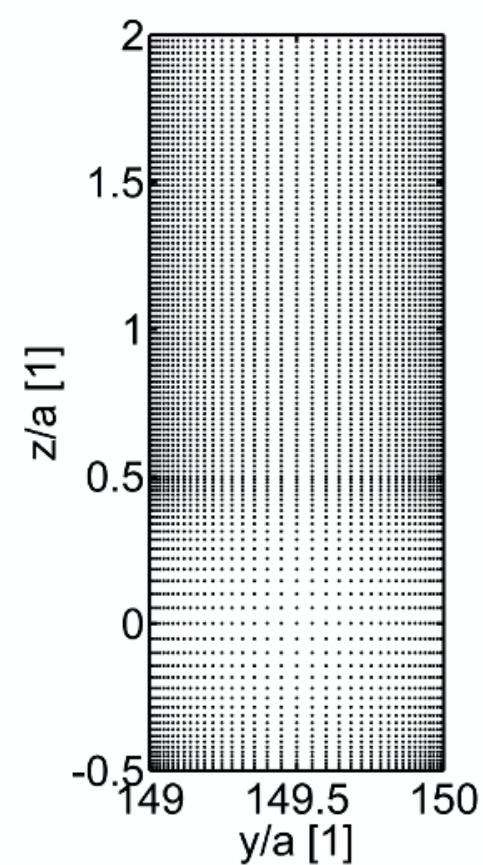

Fig. 1. Computational mesh grid distribution along/across the evaluated part of channel.

was considered and created for all 3 axis of Cartesian coordinate system, in which the geometry was created. It was the thickening of the mesh towards the wall and corners of the channel model. The density of the mesh elements was increased further in the longitudinal direction, i.e. downstream. This thickening was kept exactly to the middle of the central element (figure 1). The scheme of mesh generation was subsequently mirrored according to one of its plane, perpendicular to the flow direction. The total number of the mesh elements was around $4 \mathrm{M}$. The areas for definition of boundary conditions were also defined within the mesh generation. These were mainly the interface between particular sections. 


\subsection{Solver setup}

For the numerical simulation itself, the commercial solver ANSYS Fluent v14 was used. In the first step, the boundary conditions were defined, where a time dependent velocity profile $v(t)\left[\mathrm{m} \mathrm{s}^{-1}\right]$ at the mesh inlet was generated using the UDF (User Defined Function), which can be described by the equation

$$
v(t)=v_{s t}+v_{a m p} 2 \pi f \Delta t,
$$

where $v_{s t}\left[m s^{-1}\right]$ represented steady velocity component, vamp $\left[\mathrm{m} \mathrm{s}^{-1}\right]$ oscillating velocity component, $f[\mathrm{~Hz}]$ frequency and $\Delta t[s]$ time step of solver, which was calculated from the given frequency $f$ and necessary number of segments $n$ of one period $T[s]$

$$
T=\frac{1}{f n},
$$

where $\mathrm{n}$ is the number of segments for one period.

For the outlet boundary condition a pressure outlet was defined, for the remaining boundary areas a boundary condition with zero velocity at the wall was defined.

An interface where data were interpolated was created between the individual sections.

The flow was considered laminar, unsteady, isothermal, incompressible and viscous. A pressure based coupled solver was used with the implicit scheme of second order of accuracy for time and space discretization.

Overall number of solved periods corresponded to the sum of periods necessary for stabilization and periods for which data were evaluated. For the stabilization mode 10 periods were considered, the number of which was verified by evaluating the flow parameters for each subsequent period and by comparing with current flow parameters of the preceding period. 10 periods were selected for evaluation. Overall number of solved periods amounted to 20 and each period was divided into 720 segments, namely by $0.5^{\circ}$. For each of 7200 points several flow parameters necessary for Proper Orthogonal Decomposition (POD) were saved. These flow parameters were saved in a $2 \mathrm{D}$ plane located in the longitudinal symmetry plane interfering to section 3 and 4 .

\section{Experiment}

For POD, the results of numerical simulations will only be considered within the paper. However, this method is not limited to these results but can be also used on data obtained from the experimental solution, e.g. using the Particle Image Velocimetry method (PIV) or stereo PIV. The resultant data from the aforementioned experimental method must meet general requirements [2]. As for data acquisition, the modal decomposition procedure is similar to results of numerical simulations.

\section{Modal decomposition}

The modal analysis can decompose and analyze the time and spatial development of continuous flow on the individual basis. In this case, the modal decomposition was performed for each of the solution for $\mathrm{N}$ snapshots of data generated for aforementioned 10 amplitudes with a constant time step, which can be written as [3]

$$
\mathbf{U}=\left[\begin{array}{lll}
\mathbf{u}_{1} \ldots & \mathbf{u}_{N-1} & \mathbf{u}_{N}
\end{array}\right] .
$$

With the help of orthogonal decomposition, which can be described as a standard modal analysis technique of identifying the coherent structures, it is possible to decompose the resultant flow field to the aforementioned spatial modes $\mathbf{X}$ and corresponding time amplitude $\mathbf{T}$ [4].

$$
\mathbf{U}=\mathbf{X} \mathbf{T} .
$$

This method helps to find the most energetically significant mode with the help of diagonalization of the correlation matrix calculated from

$$
\mathbf{C}=\mathbf{U}^{T} \mathbf{W} \mathbf{U}
$$

where matrix $\mathbf{W}$ is a weight matrix, which has, in this case, the volume of cells from the examined area on its diagonal.

The evaluation of POD modes leads to solving problems of eigenvalues and eigenvectors

$$
\mathbf{C v}_{i}=\lambda_{i} \mathbf{v}_{i} \quad i=1, \ldots, N .
$$

The eigenvalues are then proportional to the energy of the given mode. The time amplitude $\mathbf{T}$ can be then obtained by scaling the norm of eigenvectors $\mathbf{v}_{i}$

$$
\mathbf{T}=\left[\mathbf{v}_{i}^{T} \sqrt{\lambda}\right]
$$

The spatial modes can be subsequently obtained by inversion (4), namely

$$
\mathbf{X}=\mathbf{U T}^{-1} \text {. }
$$

\section{Results}

For solved combinations of flow parameters listed in table 1 several regimes of pulsatile flow were evaluated with the help of POD method.

Parameters Re,Amp, $f$ and $\alpha$ in the table 1 represents $R e$ as Reynolds number for time average steady velocity defined by

$$
\operatorname{Re}(t)=\frac{\bar{v}_{s t a c}(t) a}{v},
$$

Amp as Reynolds number for time average oscillating velocity defined by

$$
\operatorname{Amp}(t)=\frac{\bar{v}_{a m p}(t) a}{v},
$$

$f$ as frequency of oscillatory flow and $\alpha$ as frequency parameter defined by

$$
\alpha=\sqrt{\frac{2 \pi f}{\eta}} \frac{a}{2},
$$

where for all above mentions parameters $v\left[\mathrm{~m}^{2} \mathrm{~s}^{-1}\right]$ represents kinematic viscosity and $\eta[\mathrm{Pas}]$ dynamic viscosity. First, the comparison of time average value of the velocity flow field of evaluated area with the first spatial mode obtained by POD analysis, which should be identical (see 
Table 1. Evaluated combinations for POD

\begin{tabular}{|c|c|c|c|c|}
\hline Label & $\operatorname{Re}[1]$ & $A m p[1]$ & $f[\mathrm{~Hz}]$ & $\alpha[1]$ \\
\hline \multicolumn{5}{|c|}{ Reference } \\
\hline XI & 600 & 300 & 0.4 & 3.96 \\
\hline \multicolumn{5}{|c|}{ Pulsatile flow } \\
\hline \multicolumn{5}{|c|}{ Influence of stationary flow } \\
\hline VII & 400 & 300 & 0.4 & 3.96 \\
\hline XI & 600 & 300 & 0.4 & 3.96 \\
\hline XIV & 800 & 300 & 0.4 & 3.96 \\
\hline \multicolumn{5}{|c|}{ Influence of oscillatory flow } \\
\hline VIII & 600 & 100 & 0.4 & 3.96 \\
\hline XI & 600 & 300 & 0.4 & 3.96 \\
\hline XIII & 600 & 500 & 0.4 & 3.96 \\
\hline \multicolumn{5}{|c|}{ Influence of frequency oscillatory flow } \\
\hline IX & 600 & 300 & 0.1 & 1.97 \\
\hline$X$ & 600 & 300 & 0.3 & 3.42 \\
\hline XII & 600 & 300 & 0.5 & 4.42 \\
\hline \multicolumn{5}{|c|}{ Stationary flow } \\
\hline I & 100 & 0 & 0 & 3.96 \\
\hline II & 400 & 0 & 0 & 3.96 \\
\hline III & 800 & 0 & 0 & 3.96 \\
\hline \multicolumn{5}{|c|}{ Oscillating flow } \\
\hline IV & 0 & 100 & 0.4 & 3.96 \\
\hline V & 0 & 300 & 0.4 & 3.96 \\
\hline VI & 0 & 500 & 0.4 & 3.96 \\
\hline
\end{tabular}

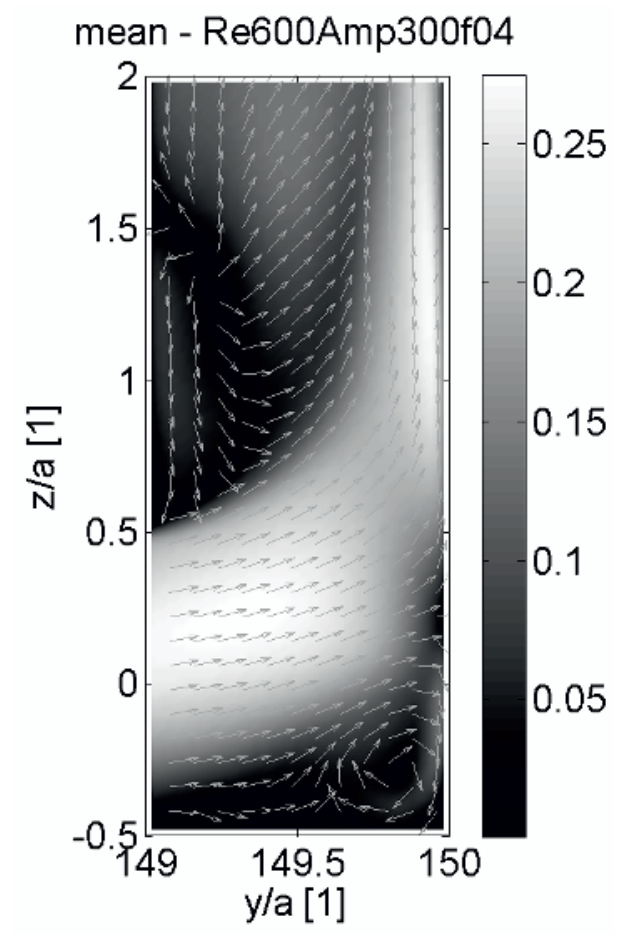

Fig. 2. Time average velocity flow field in the investigated area for case with $R e=600, A m p=300$ and $f=0.4$.

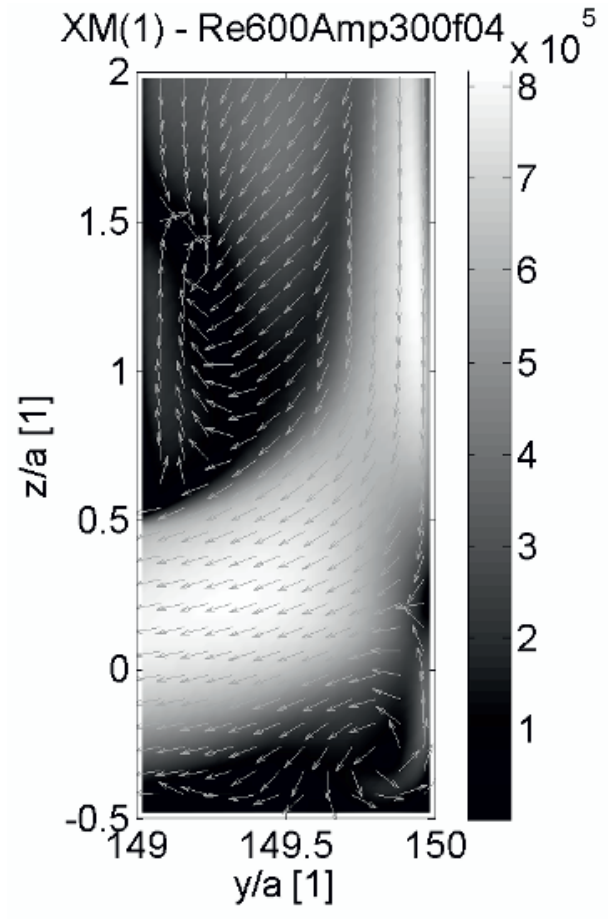

Fig. 3. First spatial mode in the investigated area for case with $R e=600, A m p=300$ and $f=0.4$.

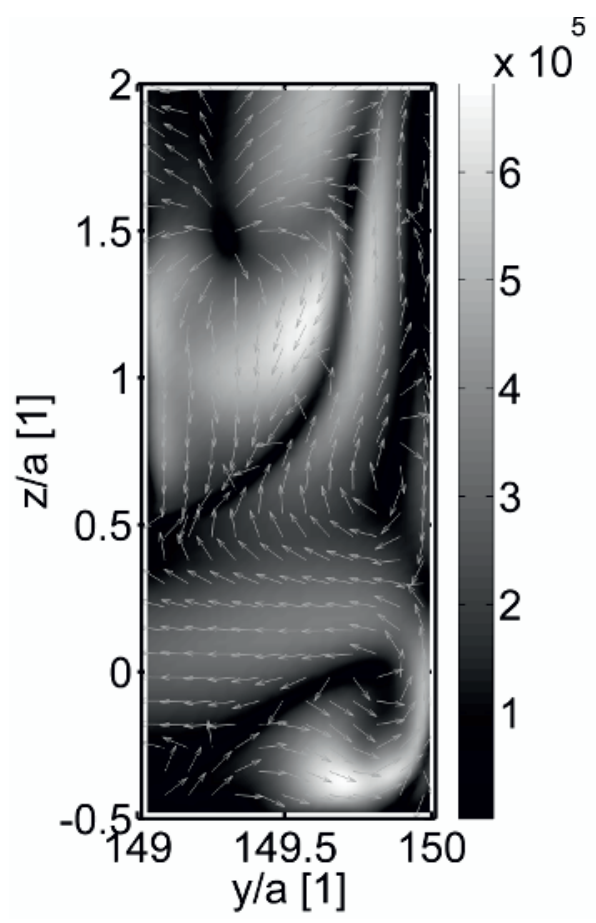

Fig. 4. Second spatial mode in the investigated area for case with $R e=600, A m p=300$ and $f=0.4$.

figure 2, figure 3). This assumption is valid for steady and pulsatile flow.

Reason why the velocity flow vectors facing backwards (see figure 3, figure 7) with compare to time average velocity flow vector (see figure 2 , figure 6 ) is in opposite sign for time amplitudes $\mathbf{T}$. From figure 4 and figure 5 can bee seen that topological structures for both spatial modes are very different. 


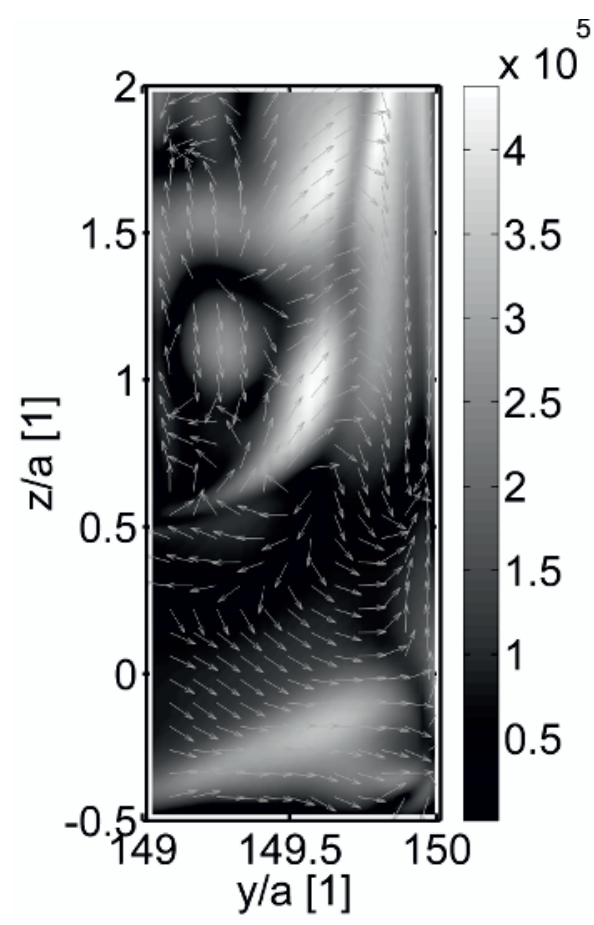

Fig. 5. Third spatial mode in the investigated area for case with $R e=600, A m p=300$ and $f=0.4$.

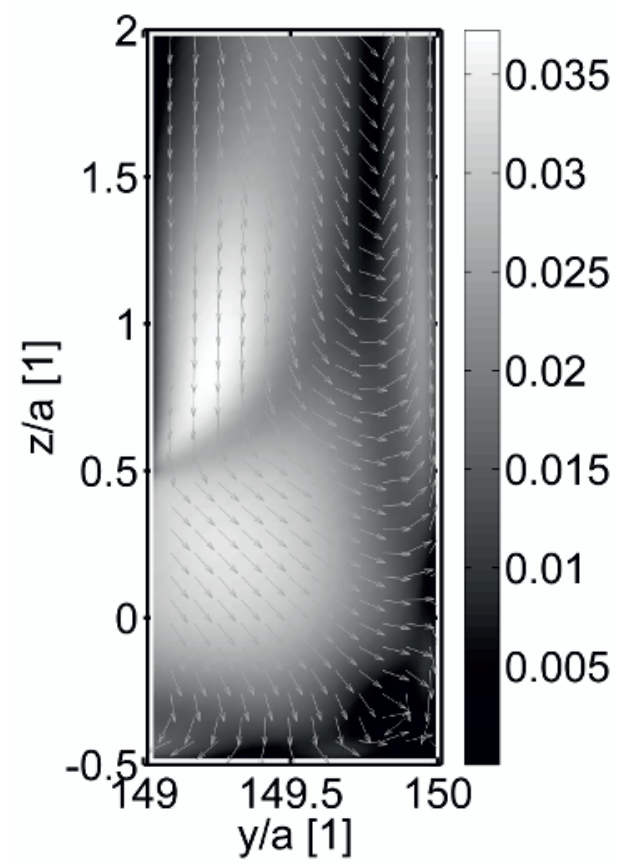

Fig. 6. Time average velocity flow field in the investigated area for case with $R e=0, A m p=300$ and $f=0.4$.

When considering only the oscillating flow where the steady velocity component was equal to zero, the second, not the first energy mode would correspond to the time average value (figure 6, figure 7). Figure 8 shows energy for first seven modes for all unsteady mode and is obvious that the most energy is in the first modes. Next six modes shows that that there are no another significant modes.

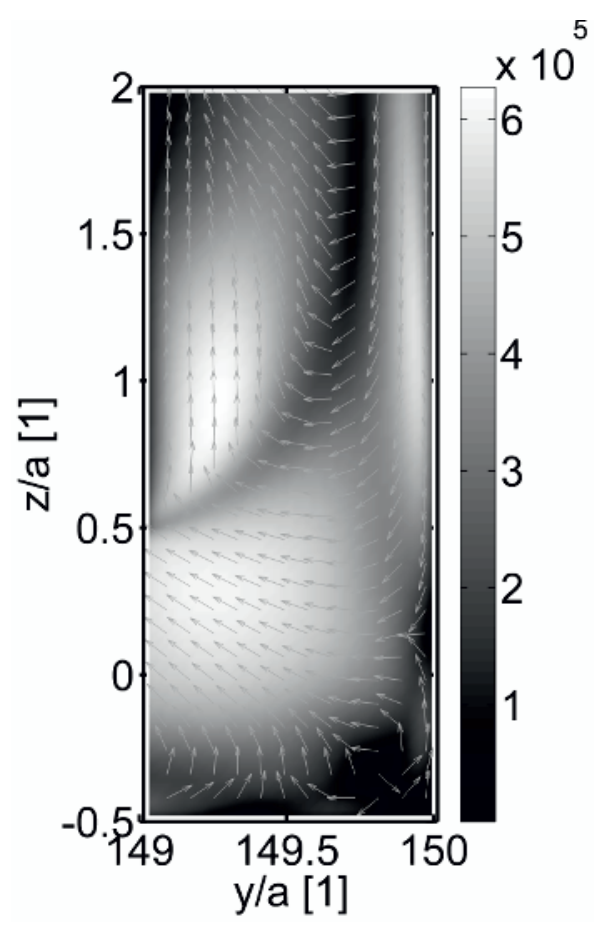

Fig. 7. Second spatial mode in the investigated area for case with $R e=0, A m p=300$ and $f=0.4$.

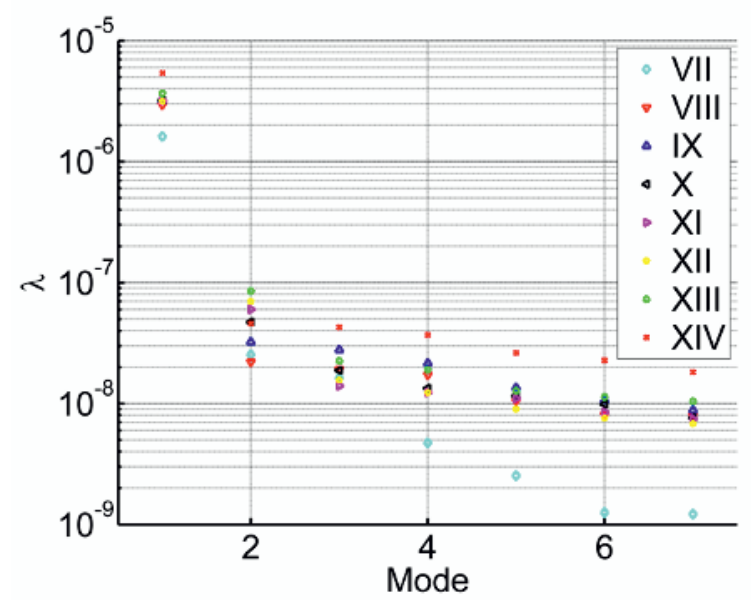

Fig. 8. Energy of POD modes for all unsteady modes (see label in table 1)

\section{Conclusion}

Within the paper, the flow field in the square cross-section channel with a $90^{\circ}$ bend for several different inlet boundary conditions was investigated with the help of numerical simulation. Subsequently, the proper orthogonal decomposition was performed on the calculated data. The paper was mainly focused on POD and determination of significant energy modes and their assignment to corresponding flow parameters. It was shown that if the flow field contains only the oscillating velocity components, then the second, not the first energy mode corresponds to the time average value of flow. In case of steady and pulsatile flow the first mode is the mode, which corresponds to the time average value of the flow field. Furthermore, it was proven that in case of pulsatile flow the course of time amplitude of first energy mode corresponds to the pulsatile flow amplitude. 


\section{Acknowledgment}

This research has been realized using the support of EU Regional Development Fund in OP R $\sharp D$ for Innovations (OP VaVpI) and Ministry for Education, Czech Republic, \# project CZ.1.05/2.1.00/03.0125 Acquisition of Technology for Vehicle Center of Sustainable Mobility. This support is gratefully acknowledged.

\section{References}

1. H. Netřebská, Dissertation, CTU in Prague, (2011)

2. J. Novotný, Journal of Flow Visualization and Image Processing, vol. 19, no. 3, (2013) p. 215-230

3. T. Hyhlík, P. Železný and, J. Čížek, EPJ Web of Conferences, Volume 45, (2013)

4. O. Frederich, D. M. Luchtenburg, Seventh International Symposium On Turbulence and Shear Flow Phenomena, (Ottawa, 2011) 